\title{
The ResultsSpace Collaborative Search Environment
}

\author{
Robert Capra, Jaime Arguello, Annie Chen, Katie Hawthorne, Gary Marchionini, Lee Shaw \\ University of North Carolina \\ School of Information and Library Science \\ Chapel Hill, NC
}

rcapra, jarguell, atchen, kathryne, march, ledshaw \{@email.unc.edu\}

\begin{abstract}
The ResultsSpace Collaborative Search Environment is a tool to support asynchronous collaborative information retrieval among a small group of collaborators. It is designed to promote awareness of collaborators' searches and the documents they have rated. Awareness is supported through several mechanisms: an area that shows a history of queries, a summary display of collaborators' ratings next to each search result, and changes in the visual salience of search results based on their aggregate rating from all collaborators. Faceted controls allow users to filter results based on specific ratings (relevant, not relevant, and maybe) and on specific collaborator(s) who have rated an item. We describe features of the system, how they are implemented, and give insights into the design rationale.
\end{abstract}

\section{Categories and Subject Descriptors}

H5.m Information interfaces and presentation; H3.3 Information search and retrieval

\section{General Terms}

Design, Human Factors.

\section{Keywords}

Collaborative search, collaborative information retrieval.

\section{INTRODUCTION}

A recent focus in information retrieval and information science communities is research on systems that can support information needs that span multiple search sessions and involve multiple people contributing to a final set of search results. The phrases "collaborative information retrieval" "collaborative information seeking" "collaborative information behavior" and "collaborative search" all refer to related work in these areas. Workshops have recently been held on these topics at JCDL 2008, CSCW 2010, GROUP 2009, GROUP 2010, ASIS\&T 2011, and CIKM 2011.

Much of the research to date has focused on synchronous collaborative search, in which people sit at different computers, but conduct searches at the same time. However, several recent surveys have shown that people do a significant amount of asynchronous collaborative searching [2][4] and that existing tools do not support this activity well [1]. Many users rely on simple "tools-at-hand" such as email and text documents to organize their collaborative searches [1]. In this project, we set out to study asynchronous collaborative search activities.

Permission to make digital or hard copies of all or part of this work for personal or classroom use is granted without fee provided that copies are not made or distributed for profit or commercial advantage and that copies bear this notice and the full citation on the first page. To copy otherwise, or republish, to post on servers or to redistribute to lists, requires prior specific permission and/or a fee.

$J C D L ' 12$, June 10-14, 2012, Washington, DC, USA.

Copyright 2012 ACM 978-1-4503-1154-0/12/06...\$10.00.
In addition, most studies of collaborative search have used tasks that asked participants to search on the open web, making computations of group precision and recall difficult (there are exceptions, e.g., [7]). The system we have developed uses an Indri search engine back-end that we can use to index specific, known corpora. To start, we have indexed news articles from the AQUAINT corpus. By using the AQUAINT corpus along with TREC tasks, we are developing an experimental system from which we can compute group-level recall and precision measures based on established tasks and relevancy ratings.

\section{RELATED SYSTEMS}

A complete review of collaborative search systems is outside the scope of this paper. However, we highlight several related systems - SearchTogether [3], CoSense [6], Coagmento [9], and Querium [8] - and refer readers to the excellent summary of collaborative search research in [5]. Prior systems have included collaborative awareness features such as query histories, integrated chat/IM, and relevance rating displays. Our system utilizes a subset of these mechanisms and explores new designs for tightly integrating collaborative awareness information within search result displays.

\section{RESULTSSPACE SYSTEM}

In this section, we outline major features of the ResultsSpace Collaborative Search Environment and describe design rationale.

Query histories - On the right side of the screen are two query history displays, one that shows collaborators' recent queries, and one that shows the user's own recent queries. Clicking on any of the queries re-issues that query and resets filters from previously active queries.

Faceted filters - Along the left side of the screen are controls that allow the user to filter results by User and Relevance Ratings.

Query display - Above the results is an area (shown in grey in Figure 1) that summarizes information about the current query including the query string and any filters that have been applied.

Relevance ratings and controls - To the left of each item in the results list, there is a set of three icons that correspond to three relevance ratings supported by the system: "relevant" = a green upwards arrow, "maybe" = a yellow box, and "not relevant" = a red downwards arrow. Users can click these icons to indicate their rating for each item or may leave an item unrated. Numbers are displayed inside each icon to show how many collaborators' have rated an item with that rating and mouse hover-over is used to show which specific collaborators made the rating. Icons with no numbers have zero ratings. This contrast between items with numbers and without helps to improve the task of scanning the list. The rating of the currently logged in user is indicated with a black bar under the rating. Figure 1 illustrates an example situation in which three collaborators (Rob, Gary, and Jaime) have been conducting searches on the topic of population growth. 
Results displays - The items in the results list are generated using Indri and consist of the article title and a snippet of text with the search terms highlighted. The presentation of items is highlighted or diminished based on the combined relevance ratings. Items with an overall positive rating are highlighted with a light green background. Items with an overall negative rating are displayed with a lighter hue font color to give them a "faded out" appearance. If an item has multiple negative ratings, it is faded out even further, making it difficult to read. Items with no ratings or with a neutral rating have no highlight or fading out. In Figure 1 , the first result has an overall positive rating (green highlight). Further down, result \#5 was rated "not relevant" by two collaborators and is faded out. Result \#6 is rated negatively, but less so than result \#5, so it is faded out to a lesser degree.

\section{SUMMARY}

The ResultsSpace Collaborative Search Environment is a system to help support and study collaborative information retrieval among small groups of collaborators. It includes collaborative awareness features including highlighting and fading out of results based on collaborators' relevance ratings. We plan to examine the effects of these awareness mechanisms to determine how they affect collaborative information retrieval including group recall, group overlap, and awareness of collaborators' actions.

\section{ACKNOWLEDGMENTS}

This work was supported by the National Science Foundation grant IIS 0812363. We thank Gene Golovchinsky and Chirag Shah for discussions related to this project.

\section{REFERENCES}

[1] Capra, R., Marchionini, G., Velasco-Martin, J., \& Muller, K. (2010). Tools-at-hand and learning in multi-session, collaborative search. In Proc. CHI 2010. ACM, New York, 951-960.
[2] Capra, R., Velasco-Martin, J. and Sams, B. (2011) Collaborative information seeking by the numbers. In Proc. of the $3^{\text {rd }}$ International Workshop on Collaborative Information Retrieval (CIR '11). ACM, New York, 7-10.

[3] Morris, M. R., \& Horvitz, E. (2007). SearchTogether: an interface for collaborative web search. In Proc. UIST 2007. ACM, New York, 3-12.

[4] Morris, M. R. (2008). A survey of collaborative web search practices. In Proc. CHI 2008. ACM, New York, 1657-1660.

[5] Morris, M. R., \& Teevan, J. (2009). Collaborative Web Search: Who, What, Where, When, and Why. Synthesis Lectures on Information Concepts, Retrieval, and Services \#14. Morgan \& Claypool.

[6] Paul, S. A., \& Morris, M. R. (2009). CoSense: enhancing sensemaking for collaborative web search. In Proc. CHI 2009. ACM, New York, 1771-1780.

[7] Pickens, J., Golovchinsky, G., Shah, C., Qvarfordt, P., \& Back, M. (2008). Algorithmic mediation for collaborative exploratory search. In Proc. SIGIR 2008. ACM, New York, 315-322.

[8] Golovchinsky, G., Diriye, A., Pickens, J. (2011). Designing for collaboration in information seeking. In Proc. Fifth Workshop on Human-Computer Interaction and Information Retrieval (HCIR 2011), Oct. 20, 2011, Mountain View, CA.

[9] Shah, C., \& Marchionini, G. (2010). Awareness in collaborative information seeking. Journal of the American Society for Information Science and Technology, 61(10), 1970-1986.

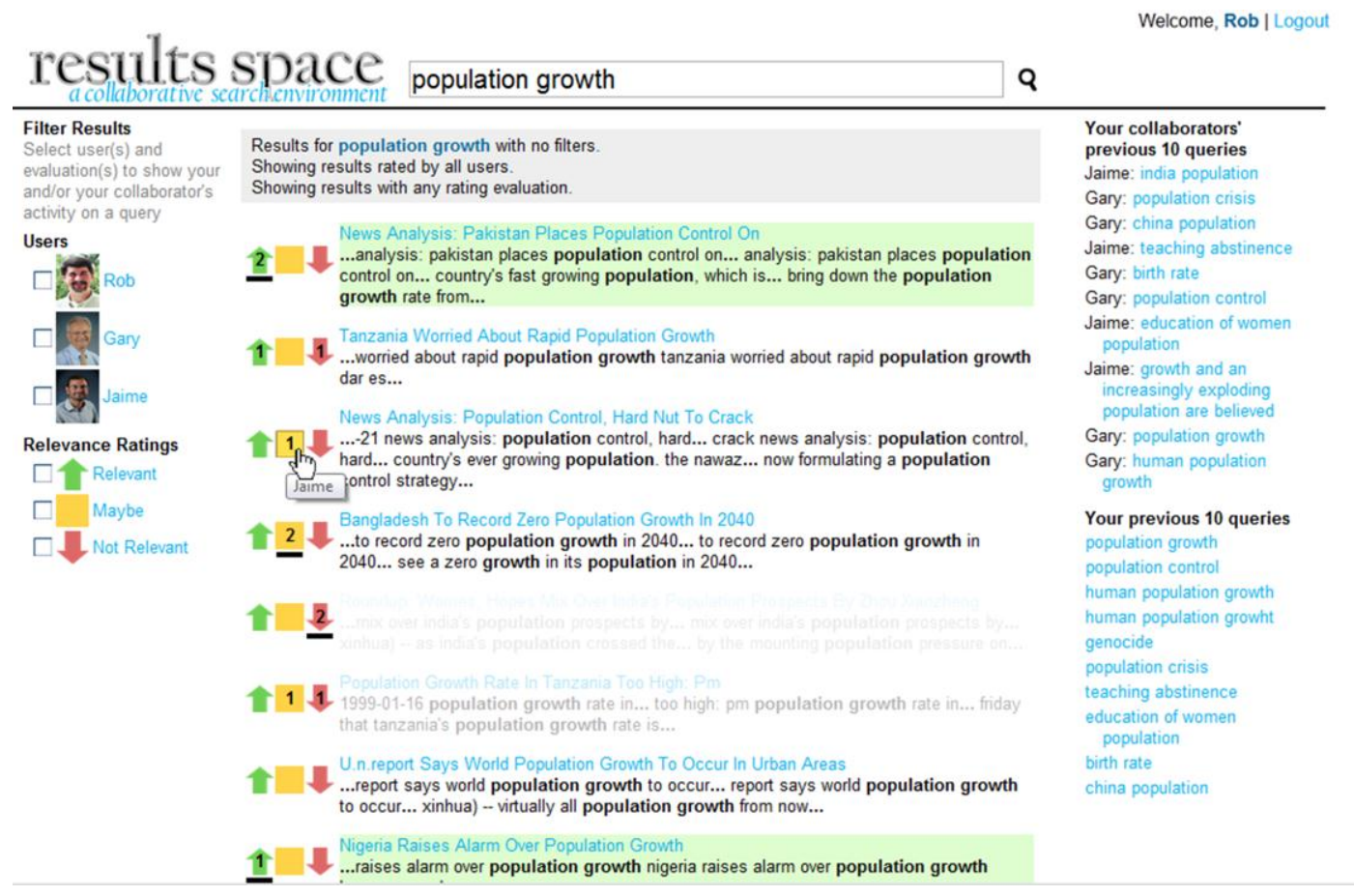

Figure 1. ResultsSpace Collaborative Search Environment 